\title{
Persistencia del desempleo regional: El caso del sur de España
}

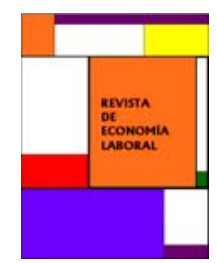

\author{
Inés P. Murillo*, Fernando Núñez y Carlos Usabiaga ${ }^{\ddagger 1}$ \\ *Universidad de Extremadura. †Universidad de Sevilla.

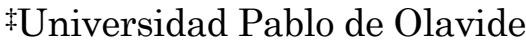

Recibido el 2 de septiembre de 2005; aceptado el 22 de marzo de 2006

Received 2 September 2005; accepted 22 March 2006

\section{Resumen:}

En el presente trabajo se aplica la metodología vector autorregresivo (VAR) propuesta por Blanchard y Katz (1992) con el objetivo de analizar la persistencia del desempleo en Andalucía y Extremadura. Los datos, trimestrales, provienen de la Encuesta de Población Activa (EPA), y corresponden al periodo 1976:3-2004:4. La principal conclusión que obtenemos es que en estas dos regiones una perturbación específica de demanda de trabajo origina efectos permanentes sobre la tasa de participación-especialmente en el caso de Andalucia-y sobre la tasa de desempleo-especialmente en el caso de Extremadura.

Palabras clave: desempleo regional, persistencia, análisis VAR.

Clasificación JEL: J40, J60, J64.

\begin{abstract}
:
In this paper we apply the autoregressive vector (VAR) methodology proposed by Blanchard $y$ Katz (1992) with the main objective of analyzing the unemployment persistence in Andalusia and Extremadura. The quarterly data are provided by the Spanish Labour Force Survey (Encuesta de Población Activa, EPA), and correspond to the 1976:3-2004:4 period. Our main conclusion is that in these two regions a specific shock in the labour demand produces permanent effects on the participation ratio-mainly in Andalusia-and on the unemployment rate-mainly in Extremadura.
\end{abstract}

Keywords: regional unemployment, persistence, VAR analysis.

JEL Classification: J40, J60, J64.

\footnotetext{
1 Autor para correspondencia: cusaiba@upo.es. Agradecemos las sugerencias recibidas de un evaluador anónimo, M.A. Malo, J.L. Raymond, J.L. Roig, J. Ramajo, A. García y F. Pedraja, así como las recibidas, respecto a versiones previas de este trabajo, en el VIII Encuentro de Economía Aplicada, las VI Jornadas de Economía Laboral y ERSA (2005). También agradecemos la financiación del Centro de Estudios Andaluces (ECOD1.05/033).
}

(C) Revista de Economía Laboral 


\section{Introducción}

El estudio del comportamiento del mercado de trabajo, y más concretamente del desempleo, ha atraído desde siempre el interés de los economistas. Sin embargo, este interés se ha visto acrecentado con las altas y persistentes tasas de desempleo observadas, especialmente en las economías europeas, en las últimas tres décadas. El análisis de ese problema se ha enfocado desde diferentes perspectivas, señalándose diversas teorías y factores explicativos: euroesclerosis, histéresis, insidersoutsiders, desempleo de larga duración, poder sindical, desajuste de cualificaciones, etc ${ }^{2}$.

El problema del desempleo no afecta con la misma intensidad a todos los países occidentales. Por el contrario, presenta una desigual incidencia, incluso cuando se consideran entornos económicos de características similares. Así, por ejemplo, para el conjunto de países integrados en la Unión Europea - exceptuando a los recién incorporados países del Este- la tasa de desempleo ha oscilado en el año 2004 entre un mínimo del $4.2 \%$ para Luxemburgo y un máximo del $10.8 \%$ para España ${ }^{3}$. Aún más llamativas resultan las diferencias de tasas de desempleo dentro de cada país, entre sus respectivas regiones ${ }^{4}$. El mercado laboral español ofrece una buena muestra de ello; así, para el año 2004, la región española situada a la cabeza de las regiones con mayor tasa de desempleo (Extremadura) alcanzaba una cifra del 17.2\%, aproximadamente tres veces la correspondiente a Navarra, la región con menor tasa de desempleo (5.54\%).

¿Qué factores explicativos están detrás de estas notables diferencias en las tasas de desempleo, tanto nacionales como regionales? Blanchard y Jimeno (1995) abordan el estudio paradigmático de los mercados de trabajo de España y Portugal, dos países con unas características políticas, económicas y sociales muy similares, pero con tasas de desempleo profundamente dispares ${ }^{5}$. La principal conclusión

\footnotetext{
${ }^{2}$ La literatura sobre el elevado desempleo europeo y español es muy amplia. Véanse, por ejemplo, desde la perspectiva europea y española respectivamente, Bean (1994) y Blanchard y Jimeno (1994).

${ }^{3}$ Véase OCDE (2005). A este respecto cabe señalar que dichas diferencias eran mucho más acusadas hace algunos años, habiéndose producido una notable reducción en las cifras del desempleo español -Bentolila y Jimeno (2003)-, parcialmente debida a motivos estadísticos.

4 Para una revisión de la literatura internacional sobre diferenciales de desempleo regionales, véase Elhorst (2000).

5 Fernández y Polo (2002) plantean un estudio análogo para el caso de Galicia y la Región Norte de Portugal.
} 
obtenida por estos autores es que no resulta sencillo identificar, en el funcionamiento y las instituciones de los respectivos mercados de trabajo, cuáles son las causas de esas diferencias en las tasas de desempleo, enfatizándose la necesidad de estudios más específicos, centrados en aspectos como la persistencia del desempleo ante las perturbaciones económicas ${ }^{6}$.

Como ya se ha señalado, Andalucía y Extremadura han mostrado, de forma permanente a lo largo de las últimas tres décadas, un notable diferencial de desempleo con respecto a la media de las regiones españolas. Diversos autores se han ocupado de estudiar esta cuestión. Así, Jimeno (1997), Herce et al. (2001) y Usabiaga (2004) abordan el análisis del diferencial de desempleo andaluz respecto al resto de España, mientras que Murillo (2000) y Prudencio (2003) plantean un estudio análogo para el caso de Extremadura. Un análisis descriptivo de ambos mercados de trabajo regionales, considerados de forma conjunta, respecto al resto de España, puede encontrarse en Murillo et al. (2004). Puede acudirse a todas estas contribuciones para un análisis pormenorizado de los aspectos más destacables de ambos mercados de trabajo. Nuestro análisis sólo pretende ampliar la evidencia disponible sobre el tema.

El presente trabajo adopta una perspectiva regional, centrándose en el estudio de los mercados laborales de Andalucía y Extremadura. Así, en la siguiente sección se estudia el grado de persistencia del desempleo en estas dos regiones, examinando para ello la respuesta de sus mercados de trabajo ante perturbaciones específicas en el empleo, siguiendo la metodología VAR (vector autorregresivo) propuesta por Blanchard y Katz (1992). Finalmente, la sección 3 recoge las principales conclusiones de nuestro trabajo.

\section{Análisis VAR de la persistencia}

Como se ha mencionado en la introducción, Blanchard y Jimeno (1995) señalan que una de las posibles vías de análisis de las diferencias en el comportamiento de los mercados laborales de entornos económicos similares es el estudio de la persistencia del desempleo. Desde la publicación del trabajo pionero de Blanchard y Katz (1992), diversos autores han estudiado la persistencia del desempleo a nivel regional7.

\footnotetext{
${ }^{6}$ A pesar de lo indicado, Blanchard y Jimeno (1995) relacionan la diferencia en la tasa de desempleo entre ambos países con el sistema de protección por desempleo y la naturaleza del proceso de desinflación seguido en las últimas décadas.

${ }^{7}$ Por ejemplo, Decressin y Fatás (1995) analizan la dinámica de los mercados de trabajo europeos a nivel regional en comparación con Estados Unidos.
} 
Para el caso español, destacan los trabajos de Jimeno y Bentolila (1998) y Mauro y Spilimbergo (1998). Jimeno y Bentolila (1998) examinan el grado de persistencia del desempleo en una región española promedio, y comparan los resultados obtenidos con los correspondientes a los mercados de trabajo regionales de Europa y Estados Unidos, concluyendo que las principales diferencias entre los mercados de trabajo locales de estos tres entornos económicos se encuentran en los efectos a largo plazo que origina una perturbación de demanda de trabajo en los mismos. En concreto, señalan que la movilidad geográfica de los trabajadores en España es mucho más reducida que en Europa y, especialmente, que en Estados Unidos. De este modo, y a diferencia de lo que ocurre en Europa y Estados Unidos, ni la tasa de participación ni la tasa de desempleo consiguen situarse en el largo plazo en los valores previos a la perturbación.

Siguiendo esta línea de estudios, en la presente sección se aborda un análisis del comportamiento de los mercados de trabajo de Andalucía y Extremadura ante perturbaciones específicas de demanda de trabajo en dichas regiones. Para ello se estima un sistema VAR utilizando datos de frecuencia trimestral relativos a la población de 16 o más años, la población activa, el empleo y el desempleo en ambas regiones y en el conjunto nacional. Estos datos, referidos al periodo 1976:03-2004:04, proceden de la Encuesta de Población Activa (EPA), que publica el Instituto Nacional de Estadística ${ }^{8}$.

El Gráfico 1 muestra las tasas de desempleo de las regiones españolas para los años 1977 y 2004, ofreciendo una primera aproximación a la persistencia del desempleo a nivel regional.

Dicho gráfico muestra la existencia de una correlación positiva entre las tasas de desempleo que presentan las distintas regiones españolas al principio y al final del periodo temporal analizado ${ }^{9}$. En este sentido, se puede afirmar que existe una cierta persistencia en la ordenación de las regiones por tasas de desempleo, siendo las regiones que

\footnotetext{
8 En concreto, las series de población con 16 o más años y de empleo proceden de la EPA obtenida con la nueva metodología de 2005. En el caso de la serie de desempleo hemos utilizado la serie ofrecida por la EPA (metodología 2002) con la nueva definición de paro establecida en el Reglamento $n^{0} 1897 / 2000$ de la Comisión Europea, de 7 de septiembre de 2000. Bajo la nueva metodología de la EPA (metodología 2005), la serie de desempleo con la nueva definición de paro sólo está disponible a partir del año 2001. El hecho de que en el tramo 2001:01-2004:04 ambas metodologías ofrezcan valores muy parecidos para el desempleo, respalda el uso de la serie histórica de desempleo obtenida con la metodología 2002. Finalmente, la serie de población activa se ha obtenido agregando las series de empleo y desempleo.

9 Jimeno y Bentolila (1998) y Mauro y Spilimbergo (1998) llegan a resultados similares en cuanto a la ordenación de las regiones por tasa de desempleo para diferentes periodos de tiempo.
} 
presentan mayores tasas de desempleo al comienzo del periodo -como Andalucía y Extremadura- las que también soportan unas cifras de desempleo más elevadas al final del mismo.

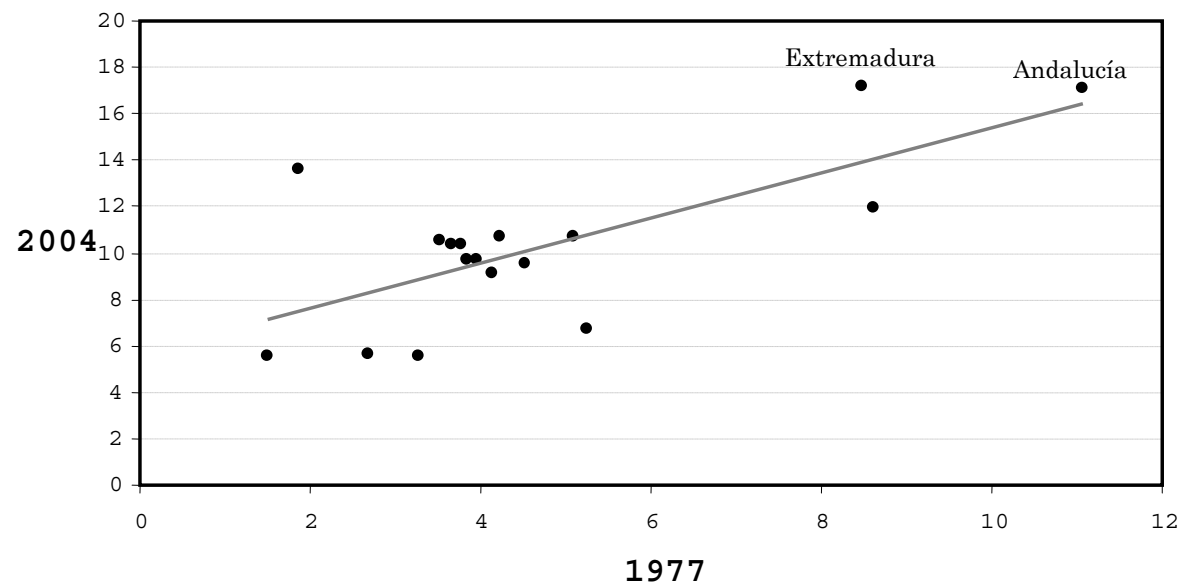

\section{Gráfico 1. Tasas de desempleo de las regiones españolas (\%)}

Para profundizar en el análisis del grado de persistencia del desempleo en estas dos regiones, a continuación se aplica la metodología propuesta por Blanchard y Katz (1992) y se estima un VAR para cada región que permite examinar cómo reaccionan sus mercados de trabajo ante una variación relativa en la tasa de crecimiento del empleo. Las variables utilizadas en el VAR son: el diferencial de la tasa de crecimiento del empleo ("e"), el diferencial de la tasa de desempleo ("d") y el diferencial de la tasa de participación ("p"). Dichos diferenciales se definen restando a los valores regionales de la variable correspondiente los del conjunto de la economía española -ambos valores expresados en logaritmos ${ }^{10}$. (1998)-:

La ecuación que se estima es la siguiente -Jimeno y Bentolila

$$
\mathrm{X}_{\mathrm{t}}=a+\beta_{1} \mathrm{X}_{\mathrm{t}-1}+\beta_{2} \mathrm{X}_{\mathrm{t}-2}+\ldots+B_{\mathrm{n}} \mathrm{X}_{\mathrm{t}-\mathrm{n}}+\varepsilon_{\mathrm{t}}
$$

donde $\mathrm{X}=(\mathrm{e}, \mathrm{d}, \mathrm{p}), \mathrm{n}$ representa el número de retardos introducidos en el modelo ${ }^{11}$ y $\varepsilon_{\mathrm{t}}$ representa el vector de innovaciones.

10 Por ejemplo, la variable "e" recoge la primera diferencia del logaritmo del empleo en Andalucía (o, en su caso, Extremadura) menos la primera diferencia del logaritmo del empleo en España.

${ }^{11}$ En la selección de retardos, los estadísticos AIC (Akaike Information Criterion) y LR (Likelihood Ratio) secuencial modificado requieren 3 retardos en la estimación del VAR 
Al estar definidas las variables de la ecuación (1) en términos relativos respecto a los valores nacionales, reflejan el comportamiento de las variables en el ámbito regional que no responde a evoluciones a nivel agregado $^{12}$. Por su parte, el primer elemento del vector de innovaciones recoge variaciones transitorias en el empleo que, siguiendo a Blanchard y Katz (1992), se interpretan como perturbaciones específicas de demanda de trabajo, y que permiten identificar la respuesta dinámica del empleo, la tasa de desempleo y la tasa de participación a variaciones en la demanda de trabajo a nivel local ${ }^{13}$. Dicho comportamiento será analizado a partir de las funciones impulso-respuesta calculadas por el método de Cholesky ${ }^{14}$.

El Gráfico 2 muestra las funciones impulso-respuesta de las variables analizadas para una desviación estándar en el crecimiento relativo del empleo. Las variables aparecen expresadas en tanto por uno, mostrando el eje de abscisas el número de trimestres considerado. En el gráfico se han incluido los intervalos de confianza asociados a cada variable en la simulación, de manera que se pueda examinar el grado de significatividad de su trayectoria a lo largo del tiempo ${ }^{15}$.

para Andalucía y 5 retardos en el caso extremeño. Por otro lado, además de las variables endógenas descritas, hemos introducido en ambas estimaciones una variable ficticia correspondiente al año 1984, la cual resulta altamente significativa. Esta dummy puede estar capturando el efecto de la entrada en vigor del subsidio para trabajadores eventuales agrarios, así como el mayor uso relativo de la contratación temporal. También hemos introducido dummies trimestrales.

12 De forma alternativa, hemos probado a estimar el modelo comparando a cada región con el resto de España -obteniendo el valor para el resto de España de las series de desempleo, empleo, población activa y población en edad de trabajar restando al valor nacional el valor de la región correspondiente. Los resultados obtenidos bajo esta variante no difieren apenas de los obtenidos empleando en la comparación los valores nacionales.

${ }^{13} \mathrm{El}$ supuesto que subyace es que la mayor parte de las variaciones en el empleo a corto plazo reflejan cambios en la demanda y no en la oferta de trabajo -Blanchard y Katz (1992), Jimeno y Bentolila (1998) y Mauro y Spilimbergo (1998).

14 Siguiendo a Jimeno y Bentolila (1998), se han calculado las funciones impulsorespuesta para el siguiente orden de las variables: "e", "d" y "p". Esta ordenación implica que las variaciones en la tasa de crecimiento del empleo se transmiten de forma contemporánea a la tasa de desempleo y a la tasa de participación, pero no a la inversa.

15 Dichos intervalos de confianza se han obtenido sumando y restando a cada respuesta al impulso dos veces su error estándar. 

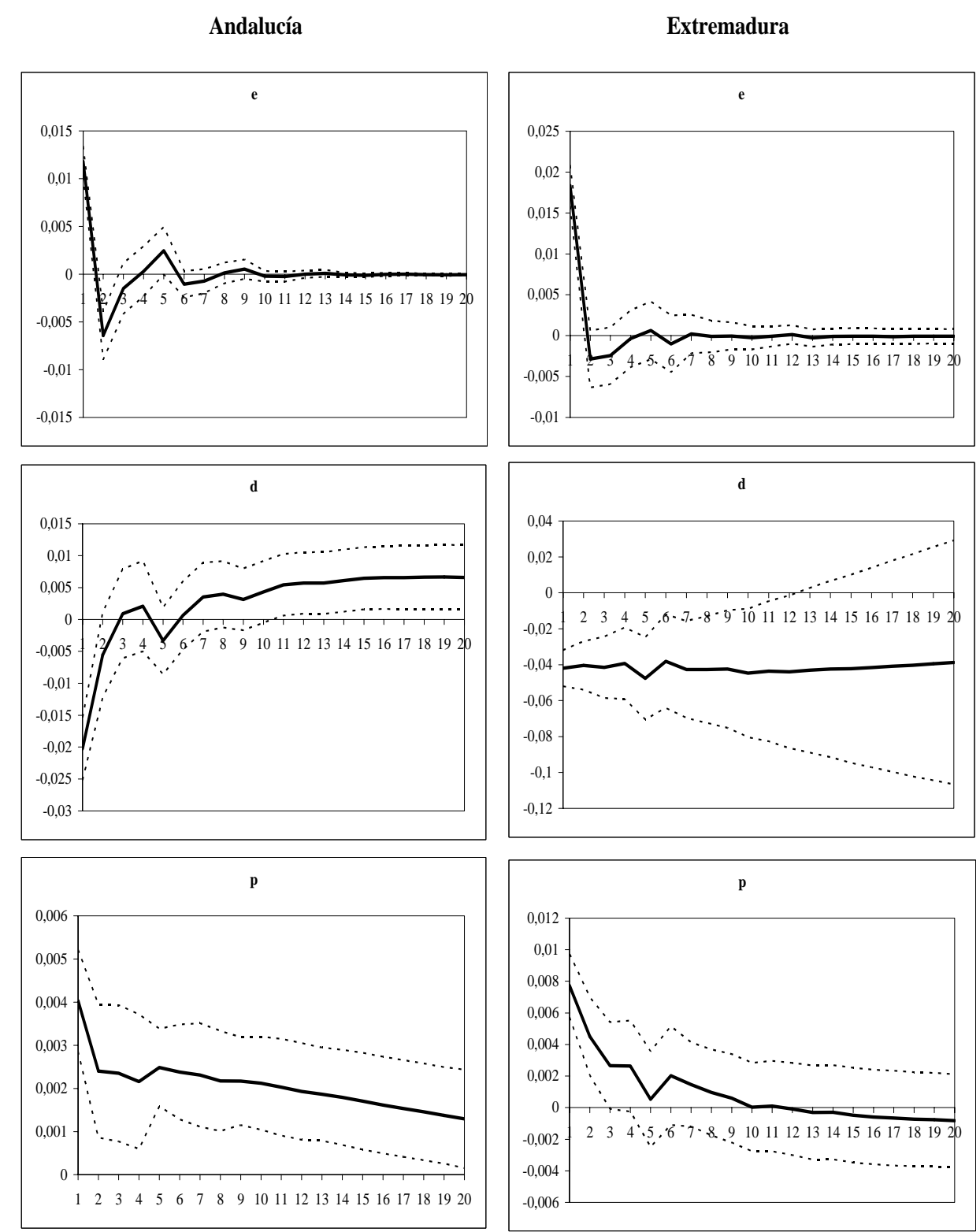

\section{Gráfico 2. Respuesta a una perturbación específica de demanda de trabajo (funciones impulso-respuesta)}

Como se observa en el gráfico, una perturbación favorable de demanda de trabajo a nivel regional, asociada a un aumento en el diferencial de la tasa de crecimiento del empleo, se traduce inicialmente en 
un aumento del diferencial de la tasa de participación y en una caída del diferencial de la tasa de desempleo en ambas regiones. Las tasas de desempleo y participación extremeñas muestran una mayor respuesta inicial ante la perturbación favorable de demanda de trabajo que las andaluzas.

En el caso del diferencial de tasa de paro, la reducción inicialmente experimentada en el caso extremeño se va absorbiendo ligeramente a largo plazo (20 trimestres), aunque sin llegar a anularse. En cambio, en el caso andaluz, la reducción inicial del diferencial pronto desaparece, por lo que los efectos beneficiosos de una perturbación favorable de demanda de trabajo son efímeros (e incluso se apunta hacia un ligero incremento del diferencial a largo plazo).

Por su parte, el incremento experimentado en ambas regiones por el diferencial de tasa de participación también se atenúa a largo plazo, llegando en el caso extremeño incluso a alcanzar ligeros valores negativos. Resulta especialmente interesante el comportamiento mostrado por el diferencial de la tasa de participación andaluza. Así, aunque el aumento que experimenta esta variable en el corto plazo no es tan acentuado como en el caso de Extremadura, el efecto a largo plazo es relativamente superior, de manera que se puede afirmar que una variación en la tasa de crecimiento del empleo en Andalucía presenta efectos permanentes sobre la tasa de participación. Este último resultado está en consonancia con las conclusiones obtenidas por Pérez et al. (2003), quienes empleando un análisis VAR para la ley de Okun concluyen que el efecto inscripción/desánimo es mayor en Andalucía que en España ${ }^{16}$, así como con las obtenidas por Murillo et al. (2004), quienes empleando la técnica de economías virtuales señalan que la elevada tasa de desempleo andaluza presenta un importante componente demográfico. Por su parte, los resultados más positivos para Extremadura de la perturbación de empleo relativo en términos de desempleo también encajan con diversos trabajos que apuntan que la principal debilidad del mercado laboral extremeño ha radicado precisamente en la falta de impulso en el empleo.

En nuestra opinión, lo más reseñable de nuestro análisis es que una variación relativa en la tasa de crecimiento del empleo en estas regiones presenta efectos permanentes sobre la tasa de desempleo especialmente en el caso de Extremadura- y sobre la tasa de participación - especialmente en el caso de Andalucía. De lo anterior se deduce que en dichas regiones la movilidad de los trabajadores juega un papel limitado

\footnotetext{
16 Los efectos inscripción y desánimo se refieren a los flujos de entrada y salida de la población activa en respuesta al ciclo económico -véanse, por ejemplo, Nickell (1987) y Blanchard (1991).
} 
como mecanismo de ajuste ante la perturbación de demanda de trabajo planteada ${ }^{17}$. Estos resultados están en consonancia con los obtenidos por Jimeno y Bentolila (1998), quienes afirman que los mercados de trabajo regionales en España presentan un menor grado de ajuste ante una perturbación de demanda de trabajo que los mercados de trabajo locales de otros entornos económicos (Europa y Estados Unidos), lo que hace que sus tasas de desempleo y participación presenten un importante grado de persistencia.

Al hilo de nuestro análisis, también podemos recordar los resultados de un análisis SVAR (vector autorregresivo estructural) de la curva de Beveridge planteado para el mercado de trabajo andaluz, en comparación con el resto de España, por Álvarez et al. (2005) ${ }^{18}$. Estos autores afirman que el predominio de las perturbaciones de reasignación frente a las perturbaciones de demanda agregada indica que el desempleo andaluz tiene un carácter más estructural que el del resto de España. Asimismo señalan que la evolución de los desempleados y las vacantes ante una perturbación de demanda agregada en Andalucía apunta hacia un problema de desajuste (o mismatch) en dicho mercado de trabajo regional.

\section{Conclusiones}

Desde la publicación del trabajo seminal de Blanchard y Katz (1992) numerosos autores han empleado el análisis VAR para analizar la persistencia del desempleo en el ámbito regional. En el presente trabajo se ha aplicado dicha metodología para analizar la persistencia del desempleo en Andalucía y Extremadura, dos regiones que han destacado en las últimas décadas por sus elevadas tasas de desempleo. La principal conclusión obtenida es que en estas dos regiones una perturbación específica de demanda de trabajo origina efectos permanentes sobre la

\footnotetext{
17 Mauro y Spilimbergo (1998) concluyen que, ante una caída en la demanda de trabajo regional en España, los trabajadores cualificados muestran una mayor tendencia a emigrar -Castillo et al. (1994) y Gámez y García Pérez (2003) llegan a la misma conclusión para el caso de Andalucía-, mientras que los trabajadores con menor nivel de estudios tienden a abandonar la población activa o a permanecer desempleados. Dado el menor nivel relativo de cualificación de la mano de obra andaluza y extremeña, éste podría ser un factor a tener en cuenta -véase también Congregado y García Pérez (2002)

18 Estos resultados también podrían extrapolarse al caso de Extremadura, ya que Dolado y Gómez (1997), en un estudio análogo planteado por grupos de regiones, agrupan a Andalucía con Extremadura y Canarias por sus características comunes a este respecto.
} 
tasa de participación -especialmente en el caso de Andalucía- y sobre la tasa de desempleo - especialmente en el caso de Extremadura-, sin que la movilidad laboral sea lo suficientemente significativa como para situar a dichas variables en los niveles previos a la perturbación. Esta evidencia complementa las conclusiones obtenidas por otros trabajos previos centrados en el análisis del diferencial de desempleo andaluz y extremeño, y que ponen su énfasis en el componente demográfico (Andalucía) y en la debilidad de la generación de empleo (Extremadura). Como ha podido apreciarse, aunque ambos mercados de trabajo comparten muchos elementos comunes, también presentan aspectos diferenciales.

\section{Bibliografía}

Álvarez, P., Núñez, F. y Usabiaga, C. (2005): "El Diferencial de Desempleo Andaluz: Análisis SVAR de la Curva de Beveridge", Centro de Estudios Andaluces, Documento de Trabajo E2005/02.

Bean, C.R. (1994): "European Unemployment: A Survey", Journal of Economic Literature, 32 (2), pp. 573-619.

Bentolila, S. y Jimeno, J.F. (2003): "Spanish Unemployment: The End of the Wild Ride?", FEDEA, Documento de Trabajo 2003-10.

Blanchard, O.J. (1991): "Wage Bargain and Unemployment Persistence", Journal of Money, Credit and Banking, 23 (2), pp. 277-292.

Blanchard, O.J. y Jimeno, J.F. (1994) (Coords.): Unemployment in Spain: Is There a Solution?, CEPR, Londres.

Blanchard, O.J. y Jimeno, J.F. (1995): "Structural Unemployment: Spain versus Portugal”, American Economic Review, 85 (2), pp. 212-218.

Blanchard, O.J. y Katz, L. (1992): "Regional Evolutions", Brookings Papers on Economic Activity, 1, pp. 1-75.

Castillo, S., Duce, R.M. y Jimeno, J.F. (1994): "El Mercado de Trabajo en Andalucía: Los Factores Determinantes del Paro Masivo y Persistente", Boletín Económico de Andalucía, 18, pp. 25-38. 
Congregado, E. y García Pérez, J.I. (2002): "El Problema del Desempleo en la Economía Andaluza (1990-2001): Análisis de la Transición desde la Educación al Mercado Laboral", Centro de Estudios Andaluces, Documento de Trabajo E2002/17.

Decressin, J. y Fatás, A. (1995): "Regional Labor Market Dynamics in Europe”, European Economic Review, 39 (9), pp. 1627-1655.

Dolado, J.J. y Gómez, R. (1997): "La Relación entre Desempleo y Vacantes en España: Perturbaciones Agregadas y de Reasignación”, Investigaciones Económicas, 21 (3), pp. 441-472.

Elhorst, J.P. (2000): "The Mystery of Regional Unemployment Differentials. A Survey of Theoretical and Empirical Explanations", University of Groningen, Research Report 00C06.

Fernández, M. y Polo, C. (2002): "Diferencias entre los Mercados de Trabajo de Dos Regiones Contiguas: el Caso de Galicia y la Región Norte de Portugal”, Revista Asturiana de Economía, 25, pp. 245-273.

Gámez, C. y García Pérez, J.I. (2003): "Flujos Migratorios de Trabajadores Andaluces (1979-1997): Un Análisis Económico con Datos Individuales", Investigaciones Regionales, 2, pp. 59-83.

Herce, J.A., Jimeno, J.F. y Usabiaga, C. (Coords.) (2001): La Economía Andaluza al Inicio del Siglo XXI. Orientaciones Estratégicas, CEA y FEDEA, Madrid.

Jimeno, J.F. (1997): "Los Factores Específicos del Paro en Andalucía", FEDEA, Documento de Trabajo 97-05.

Jimeno, J.F. y Bentolila, S. (1998): "Regional Unemployment Persistence (Spain, 1976-1994)”, Labour Economics, 5 (1), pp. 25-51.

Mauro, P. y Spilimbergo, A. (1998): "How Do The Skilled and the Unskilled Respond to Regional Shocks? The Case of Spain”, International Monetary Fund, Working Paper 98-77.

Murillo, I.P. (2000): "Mercado de Trabajo. Factores Específicos del Diferencial de Paro en Extremadura”, Universidad de Extremadura, mimeo. 
Murillo, I.P., Núñez, F. y Usabiaga, C. (2004): "Análisis del Diferencial de Desempleo Andaluz y Extremeño", Revista de Estudios Regionales, 72, pp. 45-83.

Nickell, S. (1987): "Why is the Wage Inflation in Britain so High?", Oxford Bulletin of Economics and Statistics, 49 (1), pp. 103-128.

OCDE (2005): OECD Employment Outlook, OCDE, París.

Pérez, J.J., Rodríguez, J. y Usabiaga, C. (2003): “Análisis Dinámico de la Relación entre Ciclo Económico y Ciclo del Desempleo: Una Aplicación Regional", Investigaciones Regionales, 2, pp. 141-162.

Prudencio, C.A. (2003): El Mercado de Trabajo en Regiones Atrasadas: El Caso de Extremadura, Tesis Doctoral, Universidad de Extremadura, Badajoz.

Usabiaga, C. (2004): El Diferencial de Desempleo Andaluz. Análisis Macroeconómico del Mercado de Trabajo Andaluz en Comparación con el Resto de España, Aconcagua, Sevilla. 